Keywords: breast neoplasms; false positive; interval cancer; family history; benign breast disease; mammography screening; personalised screening

\title{
Cumulative risk of breast cancer screening outcomes according to the presence of previous benign breast disease and family history of breast cancer: supporting personalised screening
}

\begin{abstract}
M Román ${ }^{1,2}$, M J Quintana ${ }^{3}$, J Ferrer ${ }^{4}, M$ Sala ${ }^{1,2}$ and X Castells ${ }^{\star, 1,2}$ on behalf of the BELE study group ${ }^{1}$ Department of Epidemiology and Evaluation, IMIM (Hospital del Mar Medical Research Institute), Passeig Marítim 25-29, Barcelona 08003, Spain; ${ }^{2}$ Health Services Research on Chronic Patients Network (REDISSEC), Barrio Labeaga s/n, Bizkaia 48960, Spain; ${ }^{3}$ Department of Epidemiology, Hospital de la Santa Creu i Sant Pau, Biomedical Research Institute Sant Pau (IIB Sant Pau), Sant Quintí 89, Barcelona 08026, Spain and ${ }^{4}$ Department of Radiology, Hospital de Santa Caterina, Dr Castany s/n, Girona 17190, Spain
\end{abstract}

Background: Our aim was to assess the cumulative risk of false-positive screening results, screen-detected cancer, and interval breast cancer in mammography screening among women with and without a previous benign breast disease and a family history of breast cancer.

Methods: The cohort included 42928 women first screened at the age of 50-51 years at three areas of the Spanish Screening Programme (Girona, and two areas in Barcelona) between 1996 and 2011, and followed up until December 2012. We used discrete-time survival models to estimate the cumulative risk of each screening outcome over 10 biennial screening exams.

Results: The cumulative risk of false-positive results, screen-detected breast cancer, and interval cancer was 36.6, 5.3, and 1.4 for women with a previous benign breast disease, $24.1,6.8$, and $1.6 \%$ for women with a family history of breast cancer, 37.9, 9.0, and $3.2 \%$; for women with both a previous benign breast disease and a family history, and 23.1, 3.2, and $0.9 \%$ for women without either of these antecedents, respectively.

Conclusions: Women with a benign breast disease or a family history of breast cancer had an increased cumulative risk of favourable and unfavourable screening outcomes than women without these characteristics. A family history of breast cancer did not increase the cumulative risk of false-positive results. Identifying different risk profiles among screening participants provides useful information to stratify women according to their individualised risk when personalised screening strategies are discussed.

Several agencies and review boards have reported evidence of reduced breast cancer mortality with mammography screening (Marmot, 2012; Lauby-Secretan et al, 2015). However, there is ongoing debate about the balance between the risk and benefits of breast cancer screening (Paci et al, 2014; Welch and Passow, 2014). Currently, population-based mammography screening programmes follow a one-size-fits-all strategy. Women within the targeted age range are screened with the same frequency,

*Correspondence: Dr X Castells; E-mail: xcastells@parcdesalutmar.cat

Received 9 December 2016; revised 23 March 2017; accepted 27 March 2017; published online 20 April 2017

(C) 2017 Cancer Research UK. All rights reserved 0007-0920/17 
mostly biennially, between the age of 50 and 69 years, independently of any risk factors or personal characteristics. Personalised breast cancer screening strategies have been proposed based on individual breast cancer risk. These strategies aim to improve the balance of benefits and harms of breast cancer screening by offering different screening frequency and modalities to invited women according to their breast cancer risk. Lately, several modelling studies have evaluated the effect of personalised breast cancer screening strategies (Schousboe et al, 2011; Vilaprinyo et al, 2014). Nevertheless, observational studies evaluating the benefits and harms of screening in women with distinct breast cancer risk profiles are scarce, and their results are still limited. Long-term assessment of the benefits and harms of women in the target population throughout their sequential participation in a screening programme is of the outmost importance to design personalised screening strategies aiming to stratify women according to their individual risks.

Benign breast disease and a family history of breast cancer are strong risk factors for breast cancer (Pharoah et al, 1997; Hartmann et $a l, 2005)$ and have been proposed as criteria for risk stratification. However, their long-term effect on breast cancer risk prediction throughout a women's screening life span and their impact on screening outcomes, such as the cumulative risk of falsepositive screening results or interval cancer, has not been evaluated together in an observational study.

We aimed to assess the cumulative risk of false-positive screening results, screen-detected cancer, and interval breast cancer in women with and without a previous benign breast disease or a family history of breast cancer who were biennially screened at the age of 50-69 years in a population-based breast cancer screening programme.

\section{MATERIALS AND METHODS}

Setting and study population. Population-based screening in Spain started in 1990 in a single setting and became nationwide in 2006. The programme has been described in detail elsewhere (Castells et al, 2006; Ascunce et al, 2010). In brief, breast cancer screening in Spain is government-funded, and follows the recommendations of the European Guidelines for Quality Assurance in Breast Cancer Screening and Diagnosis (Perry et al, 2006). The programme is organised into administrative screening settings responsible for the local application of screening in their area. Women aged 50-69 years are invited to participate in the screening programme every 2 years by written letter to a two-view mammography. Screening mammograms are interpreted by trained breast radiologists. Prior mammograms are retrieved for comparison at subsequent screens. Screening mammograms are classified according to the BI-RADS scale or equivalent (Ascunce et al, 2010). Women with abnormal mammographic findings are recalled for further assessments to confirm or rule out malignancy. Further assessments take place 1-4 weeks after the screening examination and include additional imaging, ultrasound, and invasive procedures. Women without a diagnosis of breast cancer after further assessment are referred back to routine screening, while women diagnosed with breast cancer are referred for treatment. All breast malignancies are histopathologically confirmed by trained pathologists.

The study was based on individual level data from three settings of the screening programme in Spain (Girona, and two areas in Barcelona) that routinely gather information on previous benign breast diseases and family history of breast cancer in women participating in the programme. Data for the study comprised information about the screening period 1996-2011 and follow-up until December 2012. Data were obtained from the databases of the screening centres and approval for their use was granted by the review boards of the institutions providing data. Informed consent was not required since the analyses were based on anonymised retrospective data.

We included all women first screened at age 50-51 years during the study period. The analyses included a women's first and all subsequent examinations. Women first screened at age 52 years or older were not included in the analyses, as they could not potentially benefit from 10 sequential biennial screening examinations from ages 50 to 69 years. Of the initial study population of 43192 women, we excluded 132 women because they had missing information on previous benign breast disease, 108 because of missing information on family history, and 24 because of missing information on both previous benign breast diseases and family history. This left 42928 women for analysis. Women with breast implants or a breast cancer diagnosis before their first screening exam are routinely excluded from the target population of the screening programme, and thus they were not included in the initial study population.

Definition of outcome measures. A false-positive screening result was defined as a recall for further assessment where no breast cancer was confirmed, regardless of the procedures performed (additional imaging and/or invasive procedures with a benign outcome). A screen-detected cancer was defined as breast cancer (ductal carcinoma in situ (DCIS) or invasive cancer) diagnosed as a result of further assessment due to abnormal findings on the screening mammogram interpretation. We defined an interval cancer as a breast cancer diagnosed within 24 months after a negative screening examination, with or without an invasive procedure, and before the next screening examination. Crude rates were calculated as the number of recalls for further assessments, the number of false-positive screening results, the number of screen-detected cancers, and the number of interval cancers, divided by the number of screening tests throughout the study period.

Information on previous benign breast diseases and first-degree family history of breast cancer was obtained from face-to-face interviews performed by trained professionals at the time of mammography in women invited to the screening programme for each screening participation. A woman was considered to have a history of a previous breast disease with a benign outcome if she reported a prior diagnosis of a benign breast disease. A family history of breast cancer was defined as having at least one firstdegree relative with a history of breast cancer.

Statistical analysis. We used discrete-time survival models to estimate the cumulative risks of false-positive screening results, screen-detected cancer, screen-detected breast cancer stratified by histological type (DCIS or invasive), and interval breast cancer. The women contributed data from the time of their first screening exam until censoring or end of follow-up (31 December 2011). Women were censored at the event of interest, or last screening participation, whichever came first.

The regression models included the number of screening rounds attended, previous benign breast disease, and family history of breast cancer. The number of screening rounds attended was the discrete time-scale in the regression models, covering 16 years of follow-up. Previous benign breast disease was classified as the presence or absence of a previous benign breast disease, and family history as the presence or absence of a first-degree family history of breast cancer. Because information on benign breast disease and family history was collected at each screening participation and could vary over time, they were introduced as time-changing variables in the regression models. Separate models were used to estimate the risks for each screening outcome.

Previous studies have reported that the risk of false-positive screening results is dependent on the number of screening rounds 
attended by an individual, that is, dependent censoring (Hubbard and Miglioretti, 2013). In our study population, because reattendance was lower in women with a false-positive result than in those with negative tests (Roman et al, 2011), the risk of a falsepositive screening result was adjusted for dependent censoring. This adjustment included the total number of screening rounds attended, ranging from one to eight, in addition to the standard model. Women diagnosed with a screen-detected cancer or interval breast cancer are not invited to participate in the screening programme again (censoring event). Consequently, estimation of the discrete-time survival model adjusted for dependent censoring was not relevant for screen-detected and interval cancer (Hubbard et al, 2016).

We tested interaction terms between previous benign breast disease and family history of breast cancer in all models, but found no significant interactions. The cumulative risk of each screening outcome was calculated as the risk for each screening round multiplied by the proportion of women without the event of interest until that screening round. The cumulative risk up to the previous screening round was then added. We estimated the cumulative risk of screening outcomes up to 10 biennial screening rounds in women aged 50-69 years. Because the number of women who attended more than seven screening rounds was small, we extrapolated our estimates for screening rounds 8-10, assuming

\section{Table 1. Characteristics of the study population}

\begin{tabular}{|l|c|}
\hline Women, No & 42928 \\
\hline Screens, No & 127104 \\
\hline Recalls for further assessment, No (\%) & $9075(71.4 \%)$ \\
\hline False-positive results, No (\%) & $7720(60.7 \%)$ \\
\hline Screen-detected cancers, No (\%) & $535(4.2 \%)$ \\
$\quad$ Invasive & $424(3.3 \%)$ \\
DCIS & $110(0.9 \%)$ \\
Unknown & $1(0.0 \%)$ \\
\hline Interval cancers, No (\%) & $156(1.2 \%)$ \\
\hline Screen number, No (\%) & $42928(33.8)$ \\
First & $30551(24.0)$ \\
Second & $21314(16.8)$ \\
Third & $14540(11.4)$ \\
Fourth & $9284(7.3)$ \\
Fifth & $8487(6.7)$ \\
Sixth or more & $5430(4.3)$ \\
\hline Year of screen, No (\%) & $20200(15.9)$ \\
1996-1999 & $39721(31.3)$ \\
2000-2003 & $61753(48.6)$ \\
\hline $2004-2007$ & \\
2008-2011 & \\
\hline Abbreviation: DCIS = ductal carcinoma in situ. Women first screened at the age of 50-51 \\
years.
\end{tabular}

that the risk was equal to that of the seventh round. Estimates up to the observed seventh screening round and estimates extrapolated up to the tenth screening round are presented in the tables. We calculated 95\% confidence intervals (95\% CI) for cumulative risk using the formula based on the Greenwood variance (Singer and Willett, 2003). Data were analysed using IBM SPSS Statistics version 21 (Armonk, NY, USA) and R statistical software version 3.2.3 (www.r-project.org).

\section{RESULTS}

Our study included 127104 screens from 42928 women first screened at age 50-51 years. The crude rate of recalls for further assessment was 71.4 per 1000 screens, the rate of false-positive screening results was 60.7 per 1000 screens, while the rate of screen-detected cancer and interval cancer was 4.2 and 1.2 per 1000 screens, respectively (Table 1 ).

Of the women screened, $74.7 \%(n=32062)$ had no previous benign breast disease or family history of breast cancer, $17.3 \%$ $(n=7443)$ had a previous benign breast disease, 5.3\% $(n=2265)$ had a first-degree family history of breast cancer, and $2.7 \%$ $(n=1158)$ had both, a benign breast disease and a family history of breast cancer. Women participating in the programme had an average of 3.0 screens. Almost half of screened women had three or more screens $(49.6 \%)$, and more than one-fifth had five or more screens (21.6\%; Table 2).

Table 3 shows the cumulative risk of screening outcomes up to the seventh screening round and extrapolated up to 10 screening rounds for women with and without a previous benign breast disease or a family history of breast cancer. The cumulative risk of screen-detected breast cancer and interval cancer was higher in women with than in women without previous benign breast disease or a family history. An increased cumulative risk of false-positive screening results was also found in women with a benign breast disease and in those with both a benign breast disease and a family history of breast cancer. In women without a previous benign breast disease or a family history, the cumulative risk of falsepositive screening results after 10 biennial screening exams was $23.1 \%$ (95\% CI, 22.1-24.1), the risk of screen-detected cancer was $3.2 \%$ (95\% CI, 2.6-3.7), and the risk of interval cancer was $0.9 \%$ (95\% CI, 0.6-1.1). In women with a benign breast disease and no family history the risk of false-positive screening results was $36.6 \%$ (95\% CI, 33.7-39.6), the risk of screen-detected breast cancer was $5.3 \%$ (95\% CI, 3.8-6.9), and the risk of interval cancer was $1.4 \%$ (95\% CI, $0.8-2.1)$. In women with a family history of breast cancer, the risk of false-positive screening results was $24.1 \%(95 \% \mathrm{CI}$, 20.7-27.5), that of screen-detected breast cancer was $6.8 \%$ (95\% CI, 4.0-9.5), and that of interval cancer was $1.6 \%$ (95\% CI, 0.5-2.7).

Table 2. Absolute number of women per screening round attended for each risk group profile

\begin{tabular}{|c|c|c|c|c|}
\hline $\begin{array}{l}\text { Rounds } \\
\text { attended }\end{array}$ & $\begin{array}{c}\text { Absence of benign breast } \\
\text { disease or family history of } \\
\text { breast cancer }\end{array}$ & $\begin{array}{c}\text { Benign breast disease and } \\
\text { no family history of breast } \\
\text { cancer }\end{array}$ & $\begin{array}{c}\text { Family history of breast cancer } \\
\text { and absence of benign breast } \\
\text { disease }\end{array}$ & $\begin{array}{c}\text { Benign breast disease and } \\
\text { a family history of breast } \\
\text { cancer }\end{array}$ \\
\hline 1 & 32062 & 7443 & 2265 & 1158 \\
\hline 2 & 22491 & 5565 & 1165 & 862 \\
\hline 3 & 15560 & 3983 & 806 & 606 \\
\hline 4 & 10708 & 2610 & 501 & 416 \\
\hline 5 & 6946 & 1581 & 268 & 256 \\
\hline 6 & 3959 & 863 & 139 & 145 \\
\hline 7 & 1719 & 396 & 50 & 46 \\
\hline 8 & 733 & 149 & & 20 \\
\hline Women were first screened at the age of 50-51 years; screened biennially from age 50 to 69 years. & \\
\hline
\end{tabular}


Table 3. Cumulative risk $(95 \% \mathrm{Cl})$ of breast cancer screening outcomes for women biennially screened from age $50 / 51$ years

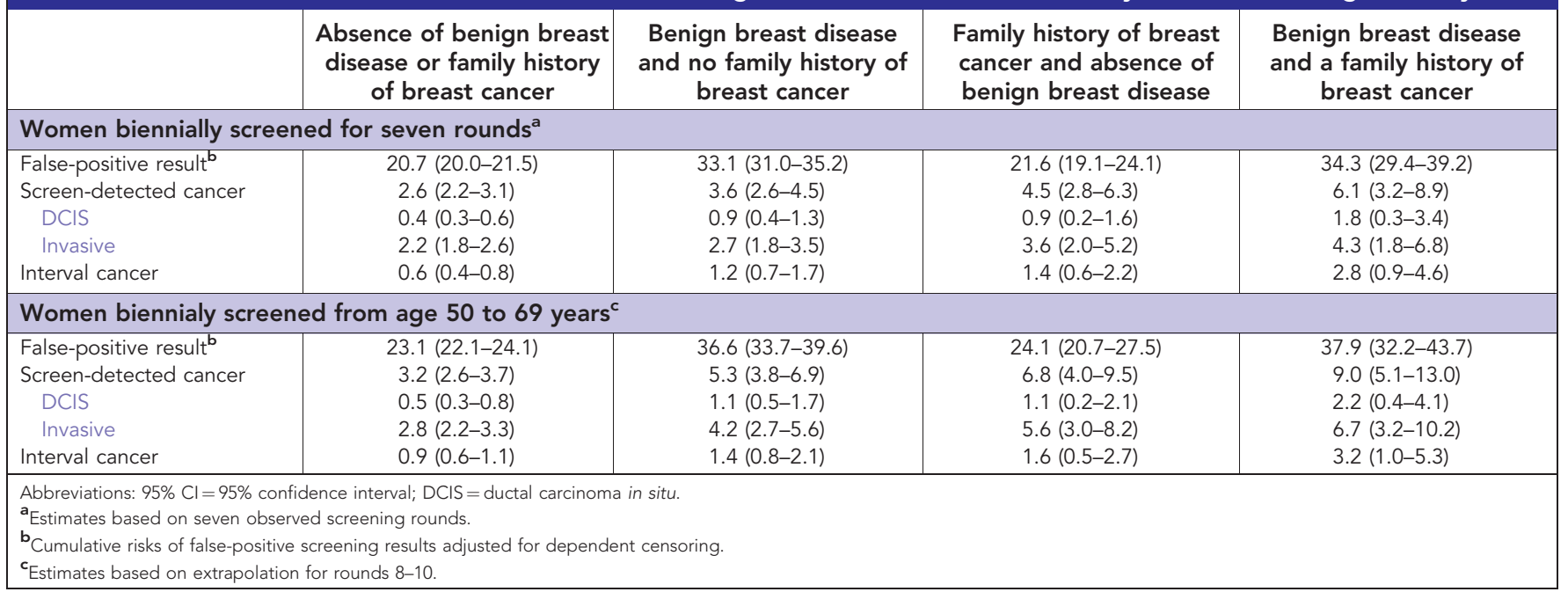
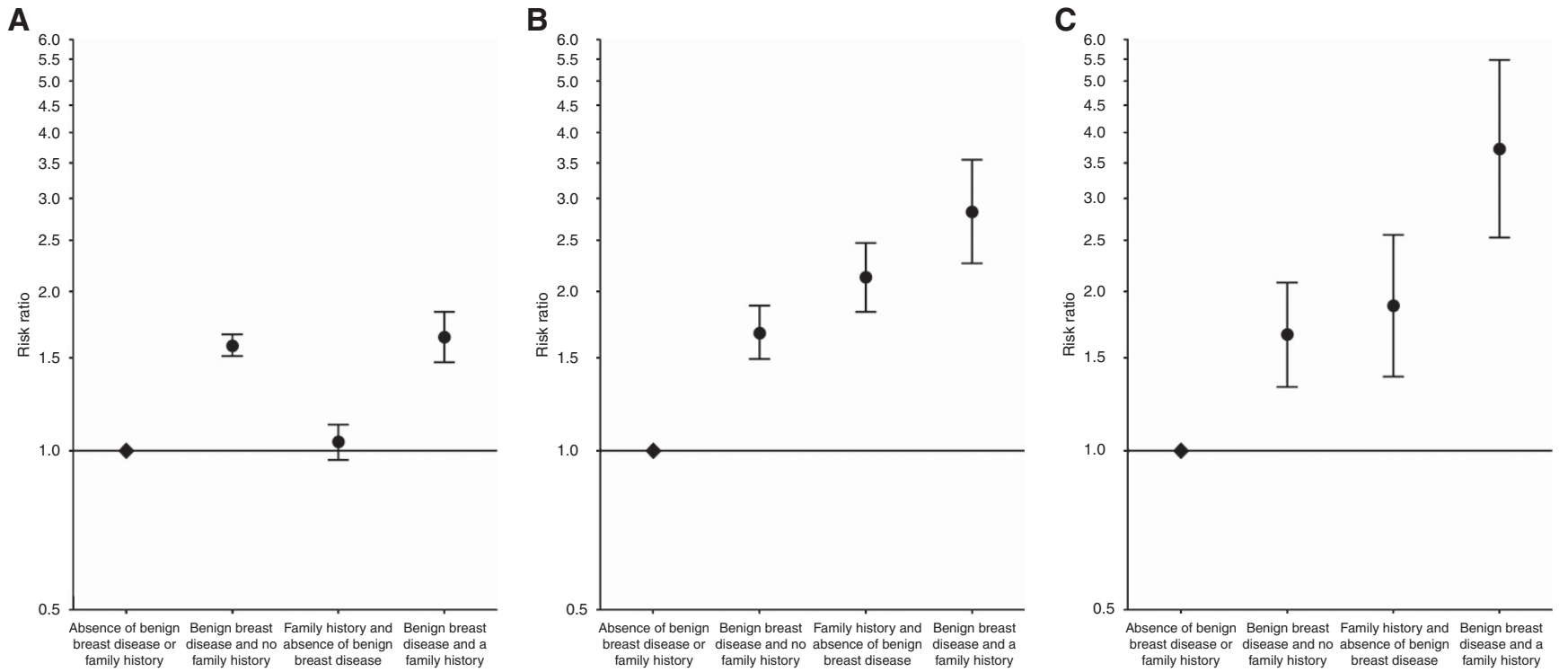

Figure 1. Risk ratios for the cumulative risk of breast cancer screening outcomes by risk profile for women biennialy screened at age $50-69$ years. Risk ratios for the cumulative risk of (A) false-positive screening results, (B) screen-detected cancer, and (C) interval breast cancer by risk profile based on the presence or absence of previous benign breast disease and family history of breast cancer, considering 'absence of benign breast disease or family history' as the reference.

Women with both a benign breast disease and a family history of breast cancer had the highest cumulative risk of screening outcomes after 10 biennial screening exams. The estimate was $37.9 \%$ (95\% CI, 32.2-43.7), 9.0\% (95\% CI, 5.1-13.0), and $3.2 \%$ (95\% CI, 1.0-5.3), for the risk of a false-positive result, screendetected cancer, and interval cancer, respectively. We also found a higher cumulative risk of DCIS and invasive breast cancer in women with than in women without a previous benign breast disease or a family history of breast cancer (Table 3).

Compared with women without a previous benign breast disease or family history, women with a benign breast disease had an increased risk of 1.6 (95\% CI, 1.5-1.7), 1.7 (95\% CI, 1.5-1.9), and 1.7 (95\% CI, 1.3-2.1) times the risk for false-positive results, screen-detected cancer, and interval breast cancer, respectively (Figure 1). Women with a family history of breast cancer had a similar risk 1.0 (95\% CI, 0.9-1.1) for a false-positive screening result, but had an increased risk of 2.1 (95\% CI, 1.8-2.5) for screendetected breast cancer, and of 1.9 (95\% CI, 1.4-2.6) for interval cancer. Women with a previous benign breast disease and a family history of breast cancer had the highest increased risk of 1.6 (95\% CI, 1.5-1.8), 2.8 (95\% CI, 2.3-3.6), and 3.7 (95\% CI, 2.5-5.5) times the risk for a false-positive result, screen-detected breast cancer, and interval cancer, respectively.

\section{DISCUSSION}

We found that women with a previous benign breast disease or a family history of breast cancer had higher cumulative risks of favourable and unfavourable screening outcomes than women without these characteristics. The risks were highest for women with both, a previous benign breast disease and a family history 
of breast cancer. The cumulative risk of false-positive results was similar for women with and without a family history of breast cancer.

This is the first study to estimate the cumulative risk of falsepositive screening results, screen-detected breast cancer, and interval cancer over a women's screening life span for women with and without a previous benign breast disease or a family history of breast cancer. There is extensive evidence that a benign breast disease and a family history increase the risk of breast cancer (Pharoah et al, 1997; Hartmann et al, 2005). However, outcome measures such as false-positive screening results and interval breast cancer have been little evaluated in women with these characteristics. Moreover, the long-term effect over 10 biennial screening exams for women with benign breast disease and a family history of breast cancer remains largely unknown. Our findings provide some first insights into the relative balance of benefits and harms of screening for women with and without a previous benign breast disease or a family history of breast cancer. A modelling study carried out in Spain, which partly used data from our study population, investigated personalised breast cancer screening strategies, including having a previous benign breast disease and a family history of breast cancer as risk factors to stratify women, together with breast density (Vilaprinyo et al, 2014). The study concluded that optimal screening was characterised by longer screening intervals (quinquennial or triennial) for women with low or moderate risk, and annual intervals for the high-risk group.

We found that women with a previous benign breast disease had a higher cumulative risk of false-positive results, screen-detected cancer, and interval cancer, whereas women with a family history of breast cancer had an increased cumulative risk of screendetected cancer and interval cancer but did not show a significantly increased cumulative risk of false-positive results. Previous studies from the USA have found a moderately increased cumulative risk of false-positive results in women with a family history of breast cancer (Hubbard et al, 2011), while a study carried out in the Netherlands found a larger effect (Ripping et al, 2016). In previously published studies from the population-based screening programme in Spain, we reported a minor elevated risk of falsepositive results in women with a family history of breast cancer (Roman et al, 2012), which is consistent with the current results. The non-increased risk found in women with a family history of breast cancer may be partially explained by the availability of prior mammograms for comparison at subsequent screens for radiologists interpreting the mammograms. In the Netherlands, radiologists also have access to prior mammograms, but their falsepositive recall rate is traditionally much lower than that of other European countries (van Luijt et al, 2013), which could emphasise the differences between family history groups. Another likely explanation is that family history in our study population may be a strong marker for breast cancer risk, but not for benign suspicious findings at mammography reading. This idea is consistent with the increased cumulative risk of breast cancer found in women with a family history of breast cancer in this and previous studies (Roman et al, 2012).

One-fourth of the study population had a previous benign breast disease or a family history of breast cancer. The impact of the increased cumulative risk of screen-detected cancer and interval breast cancer in these women translates into nearly $40 \%$ of breast malignancies (screen-detected and interval breast cancer) being diagnosed in women with at least one of these characteristics. The results of modelling studies investigating risk-based screening strategies showed that strategies based on individualised risk, including a benign breast disease and a family history of breast cancer, could improve the effectiveness of breast cancer screening (Schousboe et al, 2011; Vilaprinyo et al, 2014). Consequently, there is a need for observational studies investigating breast cancer mortality and overdiagnosis in women with a benign breast disease or a family history of breast cancer.

Previous research has indicated that if the event of interest leads to discontinuation of screening (censoring), adjustment for dependent censoring in the estimates is advisable to avoid bias (Hubbard and Miglioretti, 2013). This is likely for false positives. In our study population, women with a false-positive result had a slightly lower re-attendance rate than women with negative tests (Roman et al, 2011). We adjusted our false-positive estimates for dependent censoring by marginalising the cumulative risk over the attendance probability.

A major strength of this study is that the data were obtained from a well-established population-based screening programme with an average participation rate of $67 \%$ of invited women, and a re-attendance rate of $91.2 \%$ (Ascunce et al, 2010). We analysed information obtained from 16 years of follow-up from all women who attended their first screening exam at the age of 50-51 years, providing representativeness of the targeted population of screening and minimising selection bias. This information allowed us to provide robust estimates for the cumulative risk of screendetected cancer, interval cancer, and false-positive screening results.

However, the study has several limitations. First, none of the participants had the possibility of receiving 10 biennial screening invitations during the study period, which led us to extrapolate the estimated risk for the eighth to tenth examination. We considered the bias introduced by extrapolation to be small because previous studies have shown that risk estimates in advanced screening rounds remain stable, which minimises a possible bias (Hubbard and Miglioretti, 2013; Roman et al, 2012, 2013a). Also, because we selected only women first screened at age 50-51 years for analyses, which ensured that the dynamics of the study population were similar through subsequent screening rounds. Moreover, the rates of screen-detected cancer have remained stable for the 16 years since mammographic screening started is Spain (Roman et al, 2013b). Another limitation was that information on previous benign breast disease and family history of breast cancer was self-reported at the time of screening examination. However, the information was assessed for each screening participation and was introduced as time-changing variables in the statistical analyses, which minimised possible bias. In addition, the proportion of women with a benign breast disease or a family history of breast cancer in this study is similar to that in previous published studies (Tice et al, 2013; Ripping et $a l, 2016)$, which lends validity to our findings. Finally, the subgroup of women with a previous benign breast disease and a family history was small $(2.7 \%$ of women in the study population), which may have resulted in wider CIs for the estimates.

\section{CONCLUSION}

Our results show that, in general, women with a previous benign breast disease or a family history of breast cancer have a higher long-term risk of favourable and unfavourable screening outcomes than women without these characteristics. The risk was even higher in women with both, a previous benign breast disease and a family history of breast cancer. The results emphasise the differences in risk profiles among screening attendees, which provide useful information to stratify women according to their individualised risk. Before giving personalised screening recommendations, there is a need for further research estimating the breast cancer mortality reduction and overdiagnosis in these groups of women. 


\section{ACKNOWLEDGEMENTS}

This work was supported by Grants from Instituto de Salud Carlos III FEDER, (PI11/01296) and (PI15/00098), and from the Network for Research into Healthcare in Chronic Diseases, REDISECC (RD12/0001/0015). We acknowledge the Benign Lesion (BELE) Study Group listed here in alphabetical order and grouped by institution: (a) IMIM (Hospital Del Mar Medical Research Institute), Barcelona, Spain: Andrea Burón, Xavier Castells, Josep Maria Corominas, Ana Rodríguez-Arana, Marta Román, Maria Sala, Sònia Servitja, Isabel Torá-Rocamora, and Mar Vernet; (b) Corporació Sanitària Parc Taulí, Sabadell, Spain: Xavier Andreu and Marisa Baré; (c) Catalan Institute of Oncology, Barcelona, Spain: Llucia Benito Aracil and Carmen Vidal; (d) Hospital de la Santa Creu i Sant Pau, Barcelona, Spain: María Jesús Quintana, Margarita Posso, and Judit Solà-Roca; (e) General Directorate of Public Health, Department of Health, Government of Cantabria, Spain: Mar Sánchez; (f) Principality of Astúrias Health Service, Spain: Carmen Natal; (g) Fundació Lliga per a La Investigació i Prevenció Del Câncer, Universitat Rovira i Virgili, Tarragona, Spain: Jaume Galceran and Francina Saladié; (h) Hospital Santa Caterina, Girona, Spain: Joana Ferrer; and (i) Catalonian Cancer Strategy, Department of Health, Barcelona, Spain: Josep Alfons Espinàs.

\section{CONFLICT OF INTEREST}

The authors declare no conflict of interest.

\section{REFERENCES}

Ascunce N, Salas D, Zubizarreta R, Almazan R, Ibanez J, Ederra M (2010) Cancer screening in Spain. Ann Oncol 21(Suppl 3): iii43-iii51.

Castells X, Sala M, Ascunce N, Salas D, Zubizarreta R, Casamitjana M (2006) Descripción del cribado del cáncer en España. Proyecto DESCRIC. Madrid: Plan de Calidad para el Sistema Nacional de Salud. Ministerio de Sanidad y Consumo. Agència d'Avaluació de Tecnologia i Recerca Mèiques de Cataluña; 2007. Available from: http://www.cribadocancer.es/ images/archivos/mama/documentacion/informes_tecnicos/archivos/ InformeDESCRIC.pdf.

Hartmann LC, Sellers TA, Frost MH, Lingle WL, Degnim AC, Ghosh K, Vierkant RA, Maloney SD, Pankratz VS, Hillman DW, Suman VJ, Johnson J, Blake C, Tlsty T, Vachon CM, Melton 3rd LJ, Visscher DW (2005) Benign breast disease and the risk of breast cancer. N Engl J Med 353: 229-237.

Hubbard RA, Kerlikowske K, Flowers CI, Yankaskas BC, Zhu W, Miglioretti DL (2011) Cumulative probability of false-positive recall or biopsy recommendation after 10 years of screening mammography: a cohort study. Ann Intern Med 155: 481-492.

Hubbard RA, Miglioretti DL (2013) A semiparametric censoring bias model for estimating the cumulative risk of a false-positive screening test under dependent censoring. Biometrics 69: 245-253.

Hubbard RA, Ripping TM, Chubak J, Broeders MJM, Miglioretti DL (2016) Statistical methods for estimating the cumulative risk of screening mammography outcomes. Cancer Epidemiol Biomarkers Prev 25: 513-520.
Lauby-Secretan B, Scoccianti C, Loomis D, Benbrahim-Tallaa L, Bouvard V, Bianchini F, Straif K (2015) Breast-cancer screening-viewpoint of the IARC Working Group. N Engl J Med 372: 2353-2358.

Marmot (2012) The benefits and harms of breast cancer screening: an independent review. Lancet 380: 1778-1786.

Paci E, Broeders M, Hofvind S, Puliti D, Duffy SW (2014) European breast cancer service screening outcomes: a first balance sheet of the benefits and harms. Cancer Epidemiol Biomarkers Prev 23: 1159-1163.

Perry N, Broeders M, de Wolf C, Törnberg S, Holland R, von Karsa L (2006) European Guidelines for Quality Assurance in Breast Cancer Screening and Diagnosis. Available from: http://www.euref.org/downloads? download=24:european-guidelines-for-quality-assurance-in-breastcancer-screening-and-diagnosis-pdf.

Pharoah PD, Day NE, Duffy S, Easton DF, Ponder BA (1997) Family history and the risk of breast cancer: a systematic review and meta-analysis. Int $J$ Cancer 71: 800-809.

Ripping TM, Hubbard RA, Otten JDM, den Heeten GJ, Verbeek ALM, Broeders MJM (2016) Towards personalized screening: cumulative risk of breast cancer screening outcomes in women with and without a first-degree relative with a history of breast cancer. Int J Cancer 138: 1619-1625.

Roman M, Hubbard RA, Sebuodegard S, Miglioretti DL, Castells X, Hofvind S (2013a) The cumulative risk of false-positive results in the Norwegian Breast Cancer Screening Program: updated results. Cancer 119(22): 3952-3958.

Roman M, Rue M, Sala M, Ascunce N, Bare M, Baroja A, De la Vega M, Galceran J, Natal C, Salas D, Sanchez-Jacob M, Zubizarreta R, Castells X (2013b) Trends in detection of invasive cancer and ductal carcinoma in situ at biennial screening mammography in Spain: a retrospective cohort study. PLoS One 8: e83121.

Roman R, Sala M, De La Vega M, Natal C, Galceran J, Gonzalez-Roman I, Baroja A, Zubizarreta R, Ascunce N, Salas D, Castells X (2011) Effect of false-positives and women's characteristics on long-term adherence to breast cancer screening. Breast Cancer Res Treat 130: 543-552.

Roman R, Sala M, Salas D, Ascunce N, Zubizarreta R, Castells X (2012) Effect of protocol-related variables and women's characteristics on the cumulative false-positive risk in breast cancer screening. Ann Oncol 23: 104-111.

Schousboe JT, Kerlikowske K, Loh A, Cummings SR (2011) Personalizing mammography by breast density and other risk factors for breast cancer: analysis of health benefits and cost-effectiveness. Ann Intern Med 155: $10-20$.

Singer J, Willett JB (2003) Fitting basic discrete-time hazard models. In: Applied Longitudinal Data Analysis: Modelling Change and Event Occurrence. Oxford University Press: New York, pp 357-467.

Tice JA, O'Meara ES, Weaver DL, Vachon C, Ballard-Barbash R, Kerlikowske K (2013) Benign breast disease, mammographic breast density, and the risk of breast cancer. J Natl Cancer Inst 105: 1043-1049. van Luijt PA, Fracheboud J, Heijnsdijk EAM, den Heeten GJ, de Koning HJ (2013) Nation-wide data on screening performance during the transition to digital mammography: observations in 6 million screens. Eur J Cancer 49: 3517-3525.

Vilaprinyo E, Forne C, Carles M, Sala M, Pla R, Castells X, Domingo L, Rue M (2014) Cost-effectiveness and harm-benefit analyses of risk-based screening strategies for breast cancer. PLoS One 9: e86858.

Welch HG, Passow HJ (2014) Quantifying the benefits and harms of screening mammography. JAMA Intern Med 174: 448-454.

This work is published under the standard license to publish agreement. After 12 months the work will become freely available and the license terms will switch to a Creative Commons AttributionNonCommercial-Share Alike 4.0 Unported License. 